\title{
Performance of a Full-Duplex System in Non-Linear and Time-Varying Self-Interference Channel
}

\author{
Visa Tapio, Marko Sonkki and Markku Juntti \\ Centre for Wireless Communications \\ University of Oulu, Finland \\ Email: firstname.lastname@oulu.fi
}

\begin{abstract}
The main challenge in full-duplex transceiver design is the self-interference (SI). Analog SI isolation is performed at radio frequency (RF) signal by using an antenna design based on the characteristic modes theory and using active cancelation processing. The RF canceler is tuned using an automatic gain control (AGC) enhanced variable-step steepest descent algorithm while transmitting a data signal. The inclusion of a non-linear power amplifier does not affect the tuning performance, when realistic adjacent channel power ratio cases are considered. However, the changes in the SI channel can have a major impact on the full-duplex link due to need to re-tune the canceler repeatedly.
\end{abstract}

Index terms - Full-duplex, self-interference, link capacity, non-linearity

\section{INTRODUCTION}

Full-duplex (FD) transceivers can transmit and receive simultaneously at the same carrier frequency offering the potential to double the spectral efficiency, to reduce air interface delays, and to facilitate improved collision detection and avoidance mechanisms in content based networks. Other benefits as well as challenges in the FD technology development have been reviewed in, e.g., [1], [2], [3].

The main problem in the FD transceiver design is the self-interference (SI), i.e., the leakage of the transmit signal to the device's own receiver. Depending on the system, the SI cancelation requirement can be well over $100 \mathrm{~dB}$. In order to achieve such a high isolation levels, the SI cancelation must be done at different stages. Reviews on different SI cancelation techniques can be found, e.g., in [4], [5], [6]. In this paper, the selfinterference cancelation is performed with two techniques: antenna isolation and active cancelation at radio frequency $(\mathrm{RF})$.

The SI consists of the signal leakage from the transmit antenna port to the receive antenna port through the antenna and reflections from the environment. The powers and delays of the reflected SI components change due to the movement of the FD transceiver itself and the movements of the objects in the environment. Although there are some published measurements of a SI channel [7], [8], there are no widely accepted models for the
SI channel. We proposed a SI channel modeling based on a ray tracing approach in [9] and use it herein in Section IV-B. It allows a relatively simple way to model a multipath SI channel.

Due to the changes in the SI channel, the phase shifters and attenuators used in the active cancelation circuitry must be tunable. The usage of a gradient descent algorithm for the tuning has been proposed in [10]. Designs reported in [11], [12] also use iterative algorithms to adjust the phase and attenuation in the feed-forward paths of the analog SI cancelers. The tuning of the SI cancelation in [10], [11], [12] is done prior the full-duplex communication. Designs in [11], [12] are front end-designs where the tuning algorithms are run on a specific control unit and an additional analog-todigital (AD) conversion is needed for the SI cancelation. In our design, all the processing is performed at the baseband processing unit, no additional AD converters are needed for the SI cancelation. The tuning of the RF cancelation circuitry is done in the half-duplex (HD) mode using the transmitted data signal without the need to use a specific tuning signal, i.e., the data transmission can be continuous. Tuning is performed with the steepest descent algorithm. Automatic gain control (AGC) is used to scale the input signals of the $\mathrm{AD}$ converters to fully utilize the dynamic range of the converters.

In this work, the effect of a non-linear power amplifier on the convergence of the tuning algorithm is evaluated. The effect of the time varying SI channel on the FD link performance is also studied. The effect of the nonlinearity is measured by the adjacent channel power ratio. The cancelation performance is characterized by SI signal power attenuation.

The rest of the paper is organized as follows. The transceiver model and the SI cancelation are described in Section II, FD link capacity is discussed in Section III, SI channel model is presented in Section IV-B and numerical results are presented in Section IV. Finally, conclusions are presented in Section V. 


\section{Transceiver AND SELF-INTERFERENCE CANCELATION}

A block diagram of a full duplex transceiver is shown in Fig. 1. The two multipliers in Fig. 1 represent the up and down converting mixers. The local oscillator signals needed in the frequency conversions are not drawn to the transceiver model. Four different techniques for the SI cancelation are included in the architecture: isolation provided by the antenna, SI cancelation at RF, SI cancelation at analog baseband and digital cancelation. Only antenna isolation and RF cancelation are considered here. Antenna design is based on the characteristic modes theory [13], [14]. The same antenna model was used also in [9], [15], [16]. The RF canceler is shown in Fig. 2. It consists of a FIR filter type structure where the phase $(\phi)$ and gain values of each tap can be tuned. The delay $(\tau)$ equals the sample time of the analog-to-digital (AD) converters.

Since changes in the environment cause the SI channel to change, the SI cancelers must be tunable. The tuning of the RF canceler is done with a transmitted data signal in a half duplex mode, i.e, no FD specific training signals for the tuning are needed. A steepest descent algorithm is used for the tuning. At each iteration step, a complex coefficient vector $\mathbf{w}(k)$ is calculated as

$$
\mathbf{w}(k)=\mathbf{w}(k-1)-\frac{\mu_{i}}{M P_{\mathrm{x}}} \sum_{n=1}^{M} y_{\mathrm{rx}}(n) \mathbf{x}_{\mathrm{SI}}^{*}(n),
$$

where $k$ is the iteration index, $M$ is the number of samples per iteration, $P_{\mathrm{x}}$ is the power of the SI signal $x_{\mathrm{SI}}, y_{\mathrm{rx}}$ is the received signal and $\mu_{i}$ is the step size of the algorithm. The numerical values of $\mu_{i}$ depend on the properties of the signal as well as on the properties of the transceiver, especially the gain of the receiver, and they have been selected by simulations to allow fast convergence at the beginning and good accuracy at the end of the tuning. Vector $\mathbf{x}_{\mathrm{SI}}$ consists of samples $x_{\mathrm{SI}}(n), x_{\mathrm{SI}}(n-1) \cdots x_{\mathrm{SI}}(n-N)$ for a $N$ length SI canceler. The gains of the amplifiers and phase values for the phase shifters in Fig. 2 are calculated as the absolute values and phases of the elements of the vector $\mathbf{w}$, respectively.

During the tuning, the signal levels at the inputs of the $\mathrm{AD}$ converters are reduced. In order to utilize the full dynamic range of the $\mathrm{AD}$ converters during the tuning, AGC is used to control the level of the $\mathrm{AD}$ converters' input signals by controlling the gain of the amplifier $G_{\mathrm{RF}}$ in Fig. 1 using Algorithm 1.

$$
\begin{aligned}
& \frac{\text { Algorithm } 1}{\text { if } P_{\mathrm{rx}}>P_{t h}} \text { then } \\
& \quad G_{\mathrm{RF}}(k)=G_{\mathrm{RF}}(k-1)-2 \mathrm{~dB} \\
& \text { else if } P_{\mathrm{rx}}(k)<P_{\mathrm{rx}}(k-1)-3 \mathrm{~dB} \text { and } G_{\mathrm{RF}}<G_{\mathrm{RF}, \text { max }} \\
& \text { then } \\
& \quad G_{\mathrm{RF}}(k)=G_{\mathrm{RF}}(k-1)+2 \mathrm{~dB}
\end{aligned}
$$

\section{end if}

$P_{t h}$ is a threshold power for preventing ADC overload. It was shown in [15] that using a variable step size in the SD algorithm can improve the convergence rate. In this case, the step is chosen based on the AGC value using Algorithm 2.

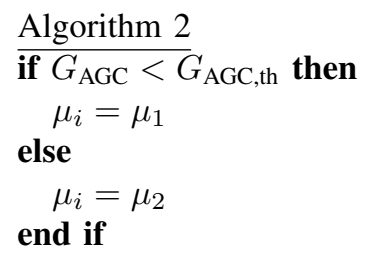

\section{Full-Duplex LINK PERFORMANCE}

The spectral efficiency of a HD link in an additive white Gaussian noise (AWGN) channel is

$$
\begin{array}{r}
R_{H D}=\alpha\left(1-\kappa_{H D}\right) \log _{2}\left(1+\gamma_{1}\right)+ \\
\quad(1-\alpha)\left(1-\kappa_{H D}\right) \log _{2}\left(1+\gamma_{2}\right),
\end{array}
$$

where $\alpha$ defines the proportion of transmission times of nodes 1 and $2, \kappa_{H D}$ is the pilot overhead of the HD link, and $\gamma_{1}, \gamma_{1}$ are the signal-to-interference-plus-noise ratios (SINR) at nodes 1 and 2, respectively, If the SINR is the same at both nodes $\left(\gamma=\gamma_{1}=\gamma_{2}\right)$, Eq. (2) reduces to

$$
R_{H D}=\left(1-\kappa_{H D}\right) \log _{2}(1+\gamma)
$$

When the nodes are operating in the FD mode, the link capacity is affected by the residual SI after the cancelation, the pilot overhead needed for the SI cancelation and the time needed to tune the RF canceler. Tuning of the $\mathrm{RF}$ canceler can be performed using a training signal or in the HD mode using the transmitted data signal for the tuning. After the tuning has stopped, the link is switched to the FD mode. Parameter $\beta \in[0,1]$ defines the proportion of time stayed in HD and FD modes. If HD mode requires the usage of additional FD specific pilots for SI channel estimation, the capacity is further reduced by the factor $\kappa_{F D}$. By taking into account of these factors the spectral efficiency of a FD link is

$$
\begin{array}{r}
R_{F D}=\beta\left(1-\kappa_{F D}-\kappa_{H D}\right)\left[\log _{2}\left(1+\gamma_{1}\right)\right. \\
\left.+\log _{2}\left(1+\gamma_{2}\right)\right]+(1-\beta) R_{H D} .
\end{array}
$$

If the tuning is done with a training signal, i.e., no data is sent during the tuning, the last term in (4) is zero. SINR terms $\left(\gamma_{1}, \gamma_{2}\right)$ include the thermal noise and residual SI after the SI cancelation. The effect of the tuning time of the RF canceler on the spectral efficiency of the FD link is shown in Fig. 3. Curves labeled with $\beta=0.9$ and $\beta=0.6$ represent cases where no data is sent during the tuning and tuning requires $10 \%$ and $40 \%$ of the transmission time, respectively. Curves labeled with ' $\beta=0.9$ data' and ' $\beta=0.6$ data' are for the cases when tuning is done in HD data transmission mode and tuning is needed during $10 \%$ and $40 \%$ of 


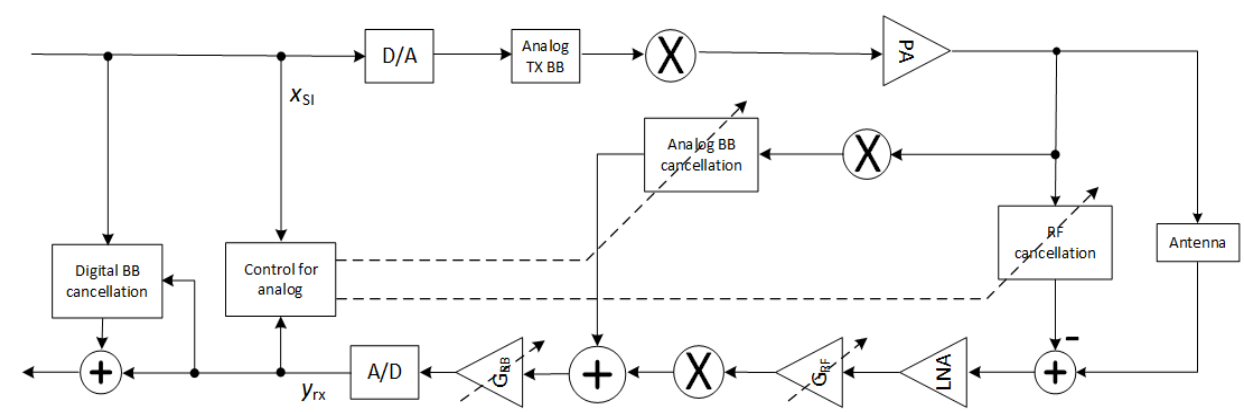

Fig. 1. FD transceiver architecture.

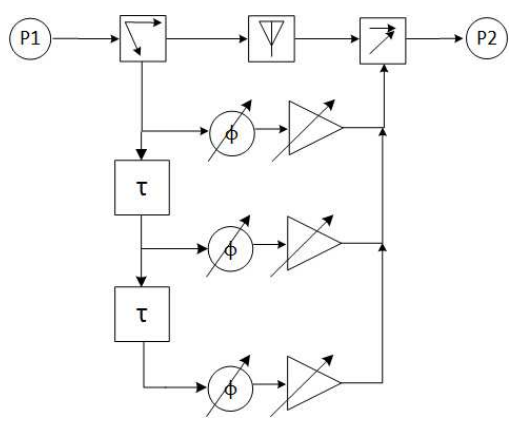

Fig. 2. Antenna and RF SI cancelation.

the transmission time, respectively. The effect of HD and FD pilot overheads are not considered in Fig. 3 $\left(\kappa_{H D}=\kappa_{F D}=0\right)$ and the SINR is assumed to be the same at both nodes $\left(\gamma=\gamma_{1}=\gamma_{2}\right)$. When the tuning of the RF canceler is needed seldom, the FD link capacity is not drastically decreased. But when the tuning is needed more frequently its effect on the capacity increases. On the other hand, when the tuning is performed while transmitting data in the HD mode, the effect of the tuning time on the link capacity is not as severe as in the case when the data transmission is stopped for the tuning.

\section{NUMERICAL EXAMPLES}

\section{A. Signal and Transceiver Model}

The signal used in the simulations is an orthogonal frequency-division multiplexing (OFDM) signal with 48 data sub-carriers and 4 pilot sub-carriers at $3.5 \mathrm{GHz}$ center frequency. Data sub-carriers are modulated using 16 level quadrature amplitude modulation (16-QAM). The bandwidth of the signal is $20 \mathrm{MHz}$. The antenna is modelled using an electromagnetic simulation tool (CST Microwave Studio). Simulated S-parameters are brought to the system model as a S-parameter file. The isolation of the antenna over the signal bandwidth is $61 \mathrm{~dB}$. The FD transceiver including the analog SI cancellers and $\mathrm{AD}$ converters is modelled using the Advanced Design System (ADS). The maximum values of the

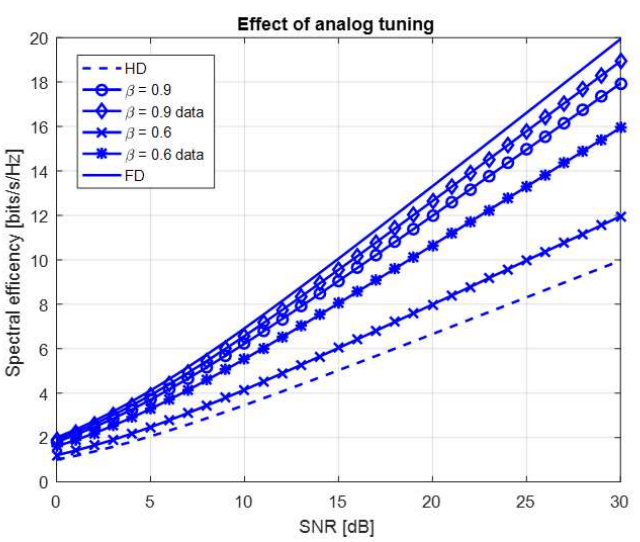

Fig. 3. FD link capacity with different FD transmission/tuning time ratios.

integral (INL) and differential (DNL) non-linearity to least significant bit of the $\mathrm{AD}$ converters are 5.0 and 0.7 , respectively. The phase and amplitude imbalance of the transceivers are $0.5^{\circ}$ and $0.1 \mathrm{~dB}$, respectively, and the phase noise data is taken from [17], [15]. The power amplifier (PA) is modeled using an non-linear amplifier model available in the Keysight's Advanced Design System (ADS) software. The amplifier non-linearity is characterized by the $1 \mathrm{~dB}$ compression point $\left(P_{1 \mathrm{dBc}}\right)$ and the output's third order intermodulation intercept point (TOI). The effect of the non-linearity on the transmitted signal is measured by the adjacent channel power ratio (ACPR). ACPR is defined as the ratio of the signal power over the $20 \mathrm{MHz}$ signal bandwidth and the leaked signal power at adjacent $20 \mathrm{MHz}$ band. The spectrum of the PA output signal is shown in Fig. 4 in the case when PA $P_{1 d B c}=24 \mathrm{dBm}$. The signal band is between the $3.49 \mathrm{GHz}$ and $3.51 \mathrm{GHz}$. The adjacent channels are bands $3.47 \mathrm{GHz}-3.49 \mathrm{GHz}$ (lower) and $3.51 \mathrm{GHz}$ $3.53 \mathrm{GHz}$ (higher). $A C P R$ is calculated as an average of the lower and higher band $A C P R$ s. The transmit power is $20 \mathrm{dBm}$ in all the cases. 


\section{B. Self-Interference Channel Model}

The SI channel consists of the direct coupling through the antenna and the reflections from the environment. In our work, reflections are modeled using a ray tracing approach [9]. In the simulations, the SI channel has 3 paths caused by the reflections in addition to the leakage through the antenna. The reflecting surfaces are assumed to be at the distances of $0.5 \mathrm{~m}, 1 \mathrm{~m}$ and $3 \mathrm{~m}$ from the transmitter. The reflection loss is assumed to be $8 \mathrm{~dB}$, and antenna the gain is $3 \mathrm{~dB}$. This gives the delay attenuation pairs $(3.33 \mathrm{~ns}, 45 \mathrm{~dB}),(6.67 \mathrm{~ns}, 50 \mathrm{~dB})$ and $(20 \mathrm{~ns}, 55 \mathrm{~dB})$ for the paths. In addition, a 180 degree phase shift is assumed for all the reflections. With these reflections the antenna isolation is decreased to $49 \mathrm{~dB}$.

\section{Numerical results}

The tuning performance of the 3-tap canceler is shown in Fig. 5. The vertical axis shows the amount of SI cancelation (SIC) in decibels (dB) provided by the canceler and the horizontal axis shows the number of iterations needed for the tuning ( $k$ in Algorithms 1 and 2). The SIC_lin curve shows the performance with a linear PA, the SIC_PA1 curve shows the performance with $P_{1 \mathrm{dBc}}=24 \mathrm{dBm}$ and the PA_SIC2 curve with $P_{1 \mathrm{dBc}}=21.8 \mathrm{dBm}$. The TOI in all the cases has been $10 \mathrm{~dB}$ above the $P_{1 \mathrm{dBc}}$. The ACPR values with these values are $-53 \mathrm{~dB},-32 \mathrm{~dB}$ and $-25 \mathrm{~dB}$, respectively. As can be seen from Fig.5, the tuning process is not affected by the non-linear operation of the PA in these cases. After the convergence the RF canceler provides $34 \mathrm{~dB}$ of additional SI attenuation $(34+49 \mathrm{~dB}$ in total). RF cancelers in [11], [12] provide about $30 \mathrm{~dB}$ of cancelation. However, it should be taken into account that the results in [11], [12] are from real measurements. Simulation results shows that the non-linearity does not have a significant effect on the tuning nor the performance of the RF SI cancelation.

The sensitivity of the RF canceler's performance on the changes in the environment were simulated by keeping the phase and attenuation values of the RF canceler fixed after the tuning and changing the delay values of the paths in the SI channel model. Firstly, the delay of the shortest path was changed from $3.33 \mathrm{~ns}$ to $3.83 \mathrm{~ns}$ in $0.01 \mathrm{~ns}$ steps (curve SIC_delay1 in Fig. 6). Secondly, the delay of all the paths were increased with $50 \mathrm{~ns}$ by $0.01 \mathrm{~ns}$ steps (curve SIC_delay2 in Fig. 6). The horizontal axis in Fig. 6, shows the number of the simulation step, i.e. the value 50 corresponds with a change in the delay by $0.5 \mathrm{~ns}(50 \cdot 0.01 \mathrm{~ns})$.

Also the sensitivity of the RF cancelation on the changes in the attenuation of SI channel's paths has been simulated (Fig. 7). The horizontal axis shows the index of the simulation step. One step corresponds in $0.1 \mathrm{~dB}$, i.e., the value 20 corresponds with $2 \mathrm{~dB}$ change. The curve SIC_dB1 shows the performance deterioration

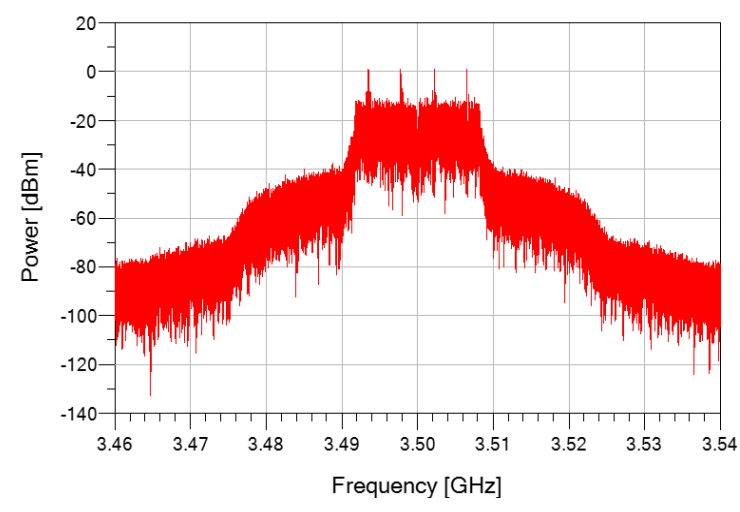

Fig. 4. Spectrum of a PA output.

when the gain of the shortest path is increased from $45 \mathrm{~dB}$ to $47 \mathrm{~dB}$ by $0.1 \mathrm{~dB}$ steps. The curve SIC_dB2 shows the effect of changing all the path gains from their original values by $0.1 \mathrm{~dB}$ steps. The results shown if Figs. 6 and 7 show the the SI cancelation is very sensitive to changes in the SI channel.

\section{CONCLUSions}

The effect of the PA non-linearity on full-duplex SI cancelation performance was evaluated. The PA was characterized with $1 \mathrm{~dB}$ compression point and the third order intermodulation intercept point. The effect of the non-linearity was measured by the adjacent channel power ratio, since it is a common performance metric used in wireless standards. The results show that the tuning with the variable step steepest descent algorithms in cooperation with automatic gain control is not sensitive to the non-linearity. The RF canceler's sensitivity to the changes in the SI channel was also evaluated by simulations. The results indicate that the SI cancelation is very sensitive to the path gain and especially to the path delay changes. When the path delay variation is in the order of half the time period of the transmitted signal, the RF canceler amplifies the SI instead of canceling it (the wave length with $3.5 \mathrm{GHz}$ center frequency is about $8.6 \mathrm{~cm}$ and the time period is about $0.29 \mathrm{~ns}$ ). The results in Figs. 6 and 7 indicate that even a slight change in the path delay and gain can require the re-tuning of the RF canceler. If the RF canceler needs to be repeatedly tuned, the HD link capacity can be compromized as discussed in Section III.

\section{ACKNOWLEDGMENT}

The research has been partially funded by Business Finland (formerly Tekes - the Finnish Funding Agency for Innovation), Nokia Oyj, Esju Oy and CoreHW This research is part of the Academy of Finland 6Genesis Flagship (grant 318927). 


\section{REFERENCES}

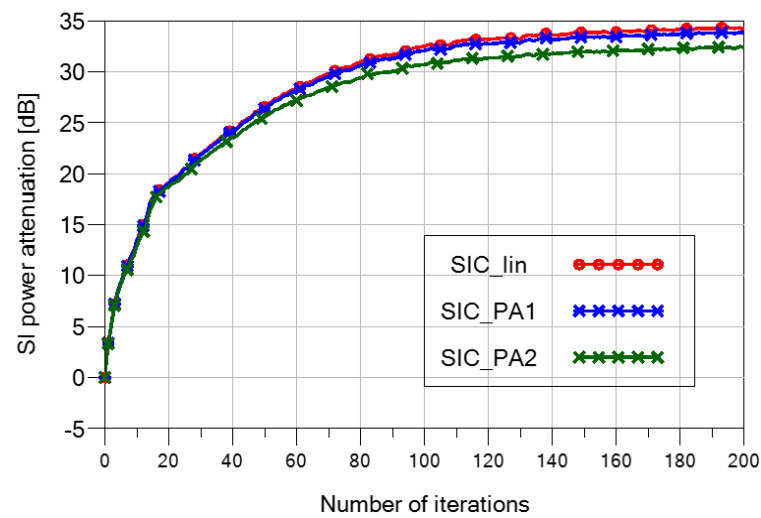

Fig. 5. Tuning performance of RF canceler.

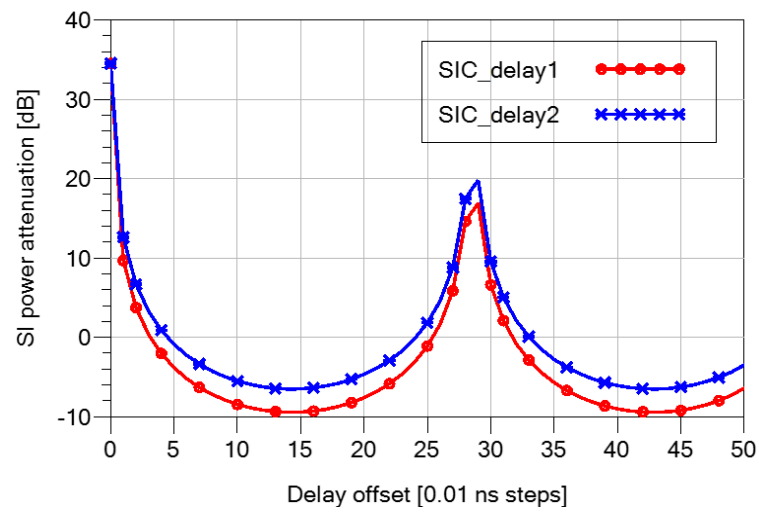

Fig. 6. Effect of the SI channel path length.

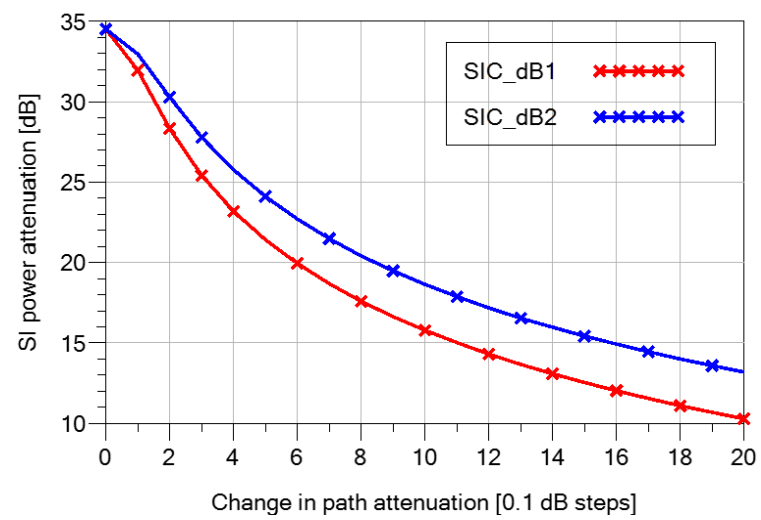

Fig. 7. Effect of the SI channel path gain.
[1] S. Hong, J. Brand, J. I. Choi, M. Jain, J. Mehlman, S. Katti, and P. Levis, "Applications of self-interference cancellation in $5 \mathrm{~g}$ and beyond," IEEE Communications Magazine, vol. 52, no. 2, pp. 114-121, February 2014

[2] A. Sabharwal, P. Schniter, D. Guo, D. W. Bliss, S. Rangarajan, and R. Wichman, "In-band full-duplex wireless: Challenges and opportunities," IEEE Journal on Selected Areas in Communications, vol. 32, no. 9, pp. 1637-1652, Sept 2014

[3] K. Rikkinen, M. Juntti, V.Tapio, A. Pouttu, B. Debaillie, C. Lavin, and M. Ghoraishi, "Full duplexing," in $5 G$ Wireless Technologies, A. Alexiou, Ed., chapter 8. IET Publishing, ISBN: 978-1-78561-061-5, 2017.

[4] Z. Zhang, X. Chai, K. Long, A. V. Vasilakos, and L. Hanzo, "Full duplex techniques for $5 \mathrm{~g}$ networks: self-interference cancellation, protocol design, and relay selection," IEEE Communications Magazine, vol. 53, no. 5, pp. 128-137, May 2015.

[5] M. Heino, D. Korpi, T. Huusari, E. Antonio-Rodriguez, S. Venkatasubramanian, T. Riihonen, L. Anttila, C. Icheln, K. Haneda, R. Wichman, and M. Valkama, "Recent advances in antenna design and interference cancellation algorithms for in-band full duplex relays," IEEE Communications Magazine, vol. 53, no. 5, pp. 91-101, May 2015.

[6] B. Debaillie, D. J. van den Broek, C. Lavn, B. van Liempd, E. A. M. Klumperink, C. Palacios, J. Craninckx, B. Nauta, and A. Prssinen, "Analog/rf solutions enabling compact full-duplex radios," IEEE Journal on Selected Areas in Communications, vol. 32, no. 9, pp. 1662-1673, Sept 2014.

[7] Kun Wang, Ruonan Zhang, Zhimeng Zhong, Xiaomei Zhang, and Xiaoyan Pang, "Measurement of self-interference channels for full-duplex relay in an urban scenario," in 2017 IEEE International Conference on Communications Workshops (ICC Workshops), 2017, pp. 1153-1158.

[8] A. Sethi, V. Tapio, and M. Juntti, "Self-interference channel for full duplex transceivers," in 2014 IEEE Wireless Communications and Networking Conference (WCNC), April 2014, pp. 781-785.

[9] V. Tapio, M. Sonkki, and M.Juntti, "Analog self-interference cancellation with automatic gain control for full-duplex transceivers," in 2018 IEEE 87th Vehicular Technology Conference: VTC2018-Spring, June 2018.

[10] M. Jain and et al., "Practical, real-time, full duplex wireless," in Proceedings of the 17th Annual International Conference on Mobile Computing and Networking (MobiCom '11), New York, NY, USA, 2011, pp. 301-312, ACM

[11] T. Huusari, Y. S. Choi, P. Liikkanen, D. Korpi, S. Talwar, and M. Valkama, "Wideband self-adaptive rf cancellation circuit for full-duplex radio: Operating principle and measurements," in 2015 IEEE 81 st Vehicular Technology Conference (VTC Spring), May 2015, pp. 1-7.

[12] K. E. Kolodziej, J. G. McMichael, and B. T. Perry, "Multitap rf canceller for in-band full-duplex wireless communications," IEEE Transactions on Wireless Communications, vol. 15, no. 6 , pp. 4321-4334, June 2016.

[13] R. Garbacz and R. Turpin, "A generalized expansion for radiated and scattered fields," IEEE Transactions on Antennas and Propagation, vol. 19, no. 3, pp. 348-358, May 1971.

[14] R. Harrington and J. Mautz, "Theory of characteristic modes for conducting bodies," IEEE Transactions on Antennas and Propagation, vol. 19, no. 5, pp. 622-628, Sep 1971.

[15] V. Tapio, M. Juntti, A. Prssinen, and K. Rikkinen, "Real time adaptive $\mathrm{rf}$ and digital self-interference cancellation for fullduplex transceivers," in 2016 50th Asilomar Conference on Signals, Systems and Computers, Nov 2016, pp. 1558-1562.

[16] V. Tapio, H. Alves, and M. Juntti, "Joint analog and digital selfinterference cancellation and full-duplex system performance," in 2017 IEEE International Conference on Acoustics, Speech and Signal Processing (ICASSP), March 2017, pp. 6553-6557.

[17] "MAX/2828/2829 Single-/Dual-Band $802.11 \mathrm{a} / \mathrm{b} / \mathrm{g}$ World-Band Transceiver ICs," Data sheet, Maxim Integrated Products, Inc. 